Société d'histoire de la révolution de 1848 et des

révolutions du XIXe siècle

Savoirs occultés : du magnétisme à l'hypnose

\title{
Une révolution manquée : le magnétisme animal dans la médecine du romantisme allemand
}

A failed revolution : the animal magnetism in the medicine of German romanticism

Luis Montiel

\section{(2) OpenEdition}

Journals

Édition électronique

URL : http://journals.openedition.org/rh19/3870

DOI : 10.4000/rh19.3870

ISSN : $1777-5329$

Éditeur

La Société de 1848

Édition imprimée

Date de publication : 1 juillet 2009

Pagination : 61-77

ISSN : 1265-1354

Référence électronique

Luis Montiel, «Une révolution manquée : le magnétisme animal dans la médecine du romantisme allemand », Revue d'histoire du XIXe siècle [En ligne], 38 | 2009, mis en ligne le 03 septembre 2009, consulté le 03 mai 2019. URL : http://journals.openedition.org/rh19/3870 ; DOI : 10.4000/rh19.3870 


\section{LUIS MONTIEL}

\section{Une révolution manquée : le magnétisme animal dans la médecine du romantisme allemand ${ }^{*}$}

Chassé par les Académies royales des sciences et de médecine françaises qui le condamnèrent en 1784, le magnétisme animal trouva dans l'Allemagne romantique un terrain propice à son développement. L'absence d'une forte centralisation, liée à la volonté de construire une nation en partant d'une culture particulière, fit qu'une médecine novatrice inventée par un Souabe, Franz Anton Mesmer, fut bientôt adoptée par la jeune génération des médecins. L'essor de ces Naturphilosophen ${ }^{2}$, bien que très contestés par les élites, parvint à faire du mesmérisme une médecine si appréciée de la population qu'elle finit par figurer pendant quelques années au programme de certaines universités allemandes. Après avoir étudié les fondements philosophiques de cette médecine, l'article montrera comment l'utilisation des thérapies magnétiques permit aux malades - particulièrement aux femmes - d'y revendiquer un rôle jusqu'à alors inexistant et de poser la question de la subjectivité de la maladie humaine.

\section{LE CÔTÉ NOCTURNE DES SCIENCES NATURELleS}

Avec sa revendication de l'unité indissoluble de la matière et de l'esprit ${ }^{3}$, la Naturphilosophie de Schelling ouvrit la porte à l'étude du "côté nocturne de la science naturelle». Par "côté nocturne», "Nachtseite», Gotthilf Heinrich von Schubert, l'un des principaux adeptes de cette philosophie, désignait les domaines de la nature qui ne pouvaient pas être explorés avec les seuls outils de la recherche scientifique, l'observation et l'expérience. Ces champs étaient donc redevables d'une autre méthodologie, basée sur la spéculation et l'ana-

1. Projet de recherche HUM2004-00777. Subdirección General de Investigación- Ministerio de Educación y Ciencia (España).

2. Philosophes de la nature.

3. «La nature doit être l'esprit visible et l'esprit la nature invisible», Friedrich Wilhelm Joseph Schelling, Ideen zu einer Philosophie der natur als Einleitung in das Studium dieser Wissenschaft, dans Werke, édition établie par Manfred Schröter, München, Beck, 1979, volume I, p. 706 (notre traduction, comme pour toutes les citations d'œuvres allemandes citées dans cet article). 
logie. La série de conférences que Schubert prononça à Dresde sur ce sujet, significativement intitulées Opinions sur le côté nocturne de la science naturelle, et publiées tout de suite en librairie ${ }^{4}$, a laissé une empreinte facile à retrouver dans l'œuvre de bien des auteurs de l'époque, tels que E.T.A. Hoffmann, Heinrich von Kleist, Achim von Arnim et Jean-Paul. Elle a défini un style de travail pour bien des partisans de la Naturphilosophie ${ }^{5}$. Schelling, pour sa part, décida de laisser à son ami Schubert le soin de développer cette branche de cette ambitieuse science "du tout", à laquelle il ne renonça jamais tout à fait, comme le montre la publication, à la mort de son épouse en 1809, de Clara, ou Du lien de la nature au monde des esprits ${ }^{6}$. Cet ouvrage, marqué par l'influence du magnétisme animal, annonce le spiritisme.

On peut assurer qu'il n'y a pas eu un Naturphilosoph qui n'ait cultivé ce Nachtseite der Naturwissenschaft pour approfondir cette dimension inconnue de la science de la nature. Le magnétisme animal, surtout après les travaux de Puységur ${ }^{7}$, semblait fournir un outil particulièrement approprié pour ce genre de recherche. Ce furent les Naturphilosophen qui essayèrent de tirer Mesmer de son exil volontaire sur les bords du lac de Constance ${ }^{8}$. Ils donnèrent ainsi au magnétisme animal l'occasion de connaitre un deuxième âge d'or loin de Paris 9. L'un des premiers d'entre eux, le physicien Johann Wilhelm Ritter, fut avec Schelling le maittre de la première génération de médecins marqués par cette philosophie durant leurs études.

Johann Wilhelm Ritter est un personnage typiquement romantique. Né à Samitz (Silésie) en 1776, il brûla frénétiquement les 33 ans de sa vie et mourut à Munich au début de 1810. L'image qui nous est parvenue de lui, parfois à travers ses propres écrits, est celle d'un possédé doué d'un tempérament volcanique souvent dirigé contre lui-même. S'il fallait le rapprocher de figures connues, il aurait pu être l'un des protagonistes des inquiétants récits d'E.T.A. Hoffmann. Son contemporain Henrik Steffens, l'un des plus fidèles disciples de Schelling, a décrit en lui un "esprit merveilleusement trouble, où l'on trouve ensemble l'obscurité et la plus éclatante clarté» ${ }^{10}$. Il faut cepen-

4. Gotthilf Heinrich von Schubert, Ansichten von der Nachtseite der Naturwissenschaften, Dresden, Arnold, 1808.

5. Jürgen Barkhoff, Magnetische Fiktionen. Literarisierung des Mesmerismus in der Romantik. Stuttgart, Metzler, 1995, p. 100.

6. Friedrich Wilhelm Joseph Schelling, Clara ou du lien de la nature au monde des esprits (Cotta, 1861), Paris, L'Herne, 1984.

7. Voir article de Jean-Pierre Peter dans ce numéro.

8. Il s'y retire après la condamnation du magnétisme par les Académies royales de médecine et des sciences françaises en 1784. L'histoire de cette renaissance a été mise au jour et systématisée par Walter Artelt, «Der Mesmerismus in Berlin ", Akademie der Wissenschaften und der Literatur in Mainz, Abhandlung des geistes- und sozialwissenschaftlicher Klasse, 1965, volume 6, p. 390-473.

9. En Allemagne le magnétisme animal arrivera à l'université - Berlin, Bonn, Tübingen - sous la plume de David Ferdinand Koreff, médecin du chancelier Prince d'Hardenberg. Voir Nils Freytag, «Der Mesmerismus auf den preussischen Gesundheitsmarkt 1812-ca. 1850», Hypnose und Kognition. Zeitschrift für die Grundlagen und klinische Anwendung von Hypnose und kognitiver Psychologie, $\mathrm{n}^{\circ} 17$, 2000, p. 124.

10. Henrik Steffens, Was ich erlebte. Aus der Erinnerung niedergeschrieben, Stuttgart-Bad Cannstatt, 
dant faire attention à l'adverbe qui précède le mot "trouble» dans cette citation du naturaliste d'origine norvégienne mais de culture allemande : cette référence au merveilleux suffit à les situer tous deux, Ritter et Steffens, dans la vague romantique. Un autre romantique plus célèbre encore - l'ami fraternel, l'âme jumelle de Ritter, Novalis -, reconnaissait à ce dernier un génie supérieur au sien propre. Dans une de ses lettres adressées à Karoline von Schlegel il demanda : «Parlez-moi donc bientôt de Ritter et Schelling. Ritter est chevalier (calembour : Ritter ist Ritter) et nous ne sommes qu'écuyers" ${ }^{11}$. Il convient de remarquer l'association des noms de Ritter et de Schelling dans ce fragment, car à ce moment-là les deux hommes se trouvaient à Iéna et y faisaient découvrir la nouvelle philosophie de la nature à une génération de jeunes passionnés.

Remarquable chercheur en physique, Ritter captivait ses auditeurs comme il devait après sa mort enchanter ses lecteurs en défendant une science symbolique, où les phénomènes observés renvoyaient toujours obligatoirement à quelque chose de caché ailleurs. Cela ne l'empêcha pas d'avoir l'intuition, en partant de la récente découverte du "galvanisme», de la notion d'électrochimie pour laquelle tout processus chimique implique un échange électrique, ni de démontrer l'existence de la radiation ultraviolette, ni de fabriquer une sorte d'accumulateur à partir de la pile de Volta, de développer les principes de l'électrolyse et enfin d'annoncer l'électromagnétisme dont l'existence sera prouvée par son élève Hans Christian Oerstedt, dix ans après sa mort. Mais, toute son œuvre, comme on l'a déjà indiqué, reste au service d'une conception de la science et de l'existence tout entière où le plus important est toujours ailleurs, dans le domaine de l'esprit ${ }^{12}$.

Dans cette perspective, la découverte, ou plutôt le postulat de l'existence d'une nouvelle force, plus subtile et universelle que l'électricité - le magnétisme - ne pouvait que devenir pour lui une stimulation intellectuelle de premier ordre. L'intérêt des médecins romantiques pour Mesmer s'accentue clairement dans les années qui encadrent la mort de Ritter. C'est précisément dans les Fragments posthumes tirés des papiers d'un jeune physicien, son œuvre la

Fromman-Holzboog, 1995, volume 2, p. 87. Dans les pages qu'il dédie à Ritter, Steffens ne cache point sa méfiance à l'égard de quelques aspects de sa personnalité, ce qui peut-être pourrait s'expliquer par le fait qu'il écrit son autobiographie dans une époque très peu "romantique». Mais il ne renie pas pour autant son admiration à son égard.

11. Roger Ayrault, La Genèse du romantisme allemand, 1797-1804, Paris, Aubier-Montaigne, 1969, 4 volumes, volume I, p. 66.

12. Gusdorf a su le voir d'une manière géniale lorsqu’il écrivit : "Considéré comme le génie même de la Naturphilosophie par Novalis et ses amis, Ritter est fort d'une science de première main, alors que Schelling compile un savoir emprunté à droite et à gauche [...] À côté de l'essai de Ritter sur le galvanisme universel (1798), l'Introduction à la première esquisse d'un système de la philosophie de la nature de Schelling (1799) donne l'impression d'un travail d'amateur; la "physique spéculative" du métaphysicien n’inspire pas la même confiance que la physique physicienne de Ritter, laquelle opère non sur des concepts, mais sur des expériences [...]. Ritter est un savant comme Reil mais Reil est médecin [...] et surtout, à la différence de Reil, Ritter est un inspiré [...]. Ritter, aussi bon physicien que les physiciens de Paris, appartient à un autre hémisphère de la pensée.", Georges Gusdorf, Le Romantisme, Paris, Payot, 1993, 2 volumes, volume 2, p. 538 et 542. 
plus célèbre, testamentaire même pour certains et pas seulement à cause de sa date de publication (en 1810, peu après la mort de l'auteur), que l'on trouve ses vues les plus personnelles, ses aperçus les plus aigus sur la nouvelle force (ou substance, selon Mesmer) naturelle. Ritter avait composé cet ensemble de manière originale. Se présentant comme le simple éditeur de fragments hérités d'un ami, il avait choisi de se distancier de son œuvre comme de sa propre biographie. Ce procédé, qui tient peut-être de l'admiration des romantiques allemands pour le Quichotte, est alors tout à fait nouveau dans le domaine de l'autobiographie et sans doute dans celui des écrits scientifiques, dont la propriété est généralement revendiquée de la façon la plus bruyante. Cependant la psychologie de Ritter n'est pas du tout celle des scientifiques actuels et sa personnalité conflictuelle semble se plaire à cette mise à distance ironique de soi-même et de sa propre œuvre. Ritter dit aimer et admirer le physicien, auteur des fragments, mais il soumet ces derniers au jugement de l'opinion publique comme quelque chose qui ne lui appartient plus, et qu'il n'a le droit d'approuver que dans son for intérieur. Heinrich Schipperges considère qu'une telle façon de faire permet à Ritter de présenter une biographie extérieure, claire et nette, basée sur des faits, tout en suggérant au lecteur sensible une autre biographie, intime, dont la lecture et la compréhension se feraient par empathie ${ }^{13}$. Ritter annonce un ensemble de fragments : de fait le lecteur trouve un texte sans unité apparente, constitué de paragraphes de longueur moyenne ou de phrases courtes, très souvent énoncées sous forme de questions. Il est vrai que le fragment est un mode d'écriture caractéristique de la littérature romantique, mais il n'en est pas moins vrai que ceux de Ritter, comme ceux de Novalis, sont ceux qui transmettent de la manière la plus évidente et même irritante pour un lecteur exigeant la sensation d'incomplétude, d'ouverture absolue, de volonté de «nescience» de vouloir savoir autrement, que l'on ne trouve que dans les poèmes de certains mystiques. Or, c'est bien de cette nature que sont les pensées que Ritter se propose de léguer à ses contemporains et à la postérité; et nous en serions de très mauvais héritiers si nous ne faisions pas l'effort de les recevoir, et aussi de les interpréter dans la mesure de nos moyens.

Commençons par sa conception de la nouvelle force, ou plutôt - car c'est ainsi qu'il semble poser lui-même la question - du nouveau territoire qui s'offre à l'examen du scientifique. Dans un de ses aphorismes denses comme le mercure, c'est précisément autour de ce nouveau venu que Ritter formule son credo philosophico-naturel :

«Dans le magnétisme animal, on sort du domaine de la volonté et on entre dans celui de l'involontaire, domaine où le corps organique se porte comme s'il

13. Heinrich Schipperges, «Nachwort» dans Johann Wilhelm Ritter, Fragmente aus dem Nachlasse eines jungen Physikers, Heidelberg, Lambert Schneider, 1969, volume 2, p. 25. 
était inorganique, en révélant ainsi les secrets des deux ordres. Là il existe une conscience qui n'a plus besoin de la volonté et de son exercice. Cette conscience involontaire se met en jeu dans le sommeil, ordinaire ou magnétique. » ${ }^{14}$

L'idée que le magnétisme animal pourrait être un médiateur entre l'homme et la nature n'est pas tout à fait nouvelle. Un des premiers magnétiseurs allemands, non encore romantique à proprement parler, Eberhard Gmelin, l'avait suggéré. Mais la manière dont Ritter pose la relation entre le monde organique et le monde inorganique est - elle - vraiment romantique. La nature extérieure à l'homme est aussi, en grande partie, organique; les plantes, par exemple, constituent une sorte de maillon entre l'animal humain et le monde minéral. Mais le propos de Ritter semble être plus radical, car à son avis c'est le corps humain qui tout à coup "se comporte comme s'il était inorganique». Ainsi le magnétisme pourrait-il fournir la clé pour comprendre l'unité de la nature. Et où trouver cette unité? Dans l'existence, que Ritter vient de découvrir, d'une "conscience de l'involontaire», façon paradoxale de nommer l'inconscient. Il s'agit d'une conscience archaïque et radicalement sage, qui agit sans intervention de la volonté et même souvent - comme le soutiendra Schubert, dont il est question dans les pages qui suivent - contre elle. Son domaine, c'est le rêve ${ }^{15}$. On remarquera que cette conception implique que le cerveau ne joue aucun rôle dans l'activité magnétique. Le centre du magnétisme humain doit être ailleurs et Ritter croit savoir où il se trouve :

«Le vrai aimant de tout corps terrestre doit être un cœur; dans le corps humain c'est le cœur qui est un aimant. Dans la terre pulse l'aimant, dans l'homme le cœur. " ${ }^{16}$

Il me semble évident que le cœur dont Ritter parle n'est pas le cœur des anatomistes, mais celui auquel l'opinion commune attribue les sentiments et les émotions. Rarement on aura mené si loin la spéculation romantique sur la voie esthétique et sentimentale; mais - il faut le dire à l'honneur des médecins qui suivront Ritter - aucun d'eux ne suivra cette voie en reniant tout ce qu'il a appris sur le cœur dans les salles de l'université. Ce que ces médecins apprécient chez Ritter et la raison pour laquelle ils en recherchent parfois même la présence physique en surmontant des obstacles considérables ${ }^{17}$, c'est qu'ils perçoivent, à juste titre, son style prophétique : il n'énonce pas des vérités établies, il annonce seulement des vérités futures. Il reviendra

14. Johann Wilhelm Ritter, Fragmente..., ouv. cité, volume 2, p. 81

15. Gotthilf Heinrich von Schubert, Die Symbolik des Traumes, Leipzig, Brockhaus, 1840, p. 19.

16. Johann Wilhelm Ritter, Fragmente..., ouv. cité, volume 2, p. 12.

17. Comme on va le lire plus loin pour Schubert, qui voyage toute une nuit de Leipzig à Iéna pour le rencontrer. 
à d'autres, «ses écuyers» selon le mot de Novalis ou ses disciples, de les mettre au jour sous leur aspect le plus concret. Et même si ces médecins conservent au muscle cardiaque le statut que lui attribue la médecine académique, je suis convaincu que ce qui les attire le plus dans la doctrine de Ritter est la notion, non-universitaire celle-ci, de "médecine de cœur». Dans cette médecine qui est encore à naître, la "découverte de l'inconscient", pour paraphraser Henri F. Ellenberger ${ }^{18}$, occupe une position majeure. D'autant plus que pour le cas de Ritter cette découverte n'est pas du tout inconsciente, ainsi qu'il l'exprime dans une lettre adressée à l'un des philosophes romantiques de la première heure, Franz von Baader :

"Je crois avoir fait une découverte d'importance, celle d'une conscience passive, de l'involontaire [...] Bien des choses ne s'expliquent qu'ainsi : l'amitié, l'amour [...] la puissance de l'imagination [...] Toutes nos actions sont de l'espèce du somnambulisme, c'est-à-dire, des réponses à des questions; et c'est nous qui interrogeons. Chacun porte en lui sa somnambule (?), dont il est lui-même le magnétiseur [...]. Dieu dans le cour : ce phénomène est absolument somnambulique. ${ }^{19}$

«Chacun porte en lui sa somnambule, dont il est le magnétiseur». Est-il possible d'énoncer d'une manière plus exacte, avec les ingrédients conceptuels fournis par l'époque, la foudroyante découverte qu'il y a quelque chose en delà - ou en deçà - de la conscience? Et n'est-il pas vrai que la «troisième révolution copernicienne», réalisée par Freud décrétant que le moi n’est pas le maître de la maison, a commencé à se produire avec cette reconnaissance que «toutes nos actions sont de l'espèce du somnambulisme»?

\section{LE BAISER DU MONDE ENTIER}

Gotthilf Heinrich von Schubert, disciple de Ritter, se situe dans le sillage de sa pensée lorsqu'il publie ses Ansichten von der Nachtseite de Naturwissenschaften, dont il a été question au début de ce travail. L'ouvrage résulte d'un cycle de conférences, ou «leçons» prononcées à Dresde en 1808. La «leçon 13» traite «du magnétisme animal et de quelques phénomènes semblables».

Tout en recommandant à ses contemporains la prudence bien particulière qu'il s'impose à lui-même, Schubert, dans cette leçon, invite ses lecteurs à sortir du cercle étroit de leur vie quotidienne et à se souvenir de ce qu'il

18. Henri F. Ellenberger, The Discovery of the Unconscious, New York, Basic Books, 1970, traduction française $A$ la découverte de l'inconscient. Histoire de la psychiatrie dynamique, Villeurbanne, SIMEP éditions, 1974 (réédition Paris, Fayard, 1994, sous le titre Histoire de la découverte de l'inconscient).

19. Albert Béguin, L'áme romantique et le rêve. Essai sur le romantisme allemand et la poésie française. Paris, José Corti, 1939. 
a exposé dans les leçons précédentes : à savoir l'existence de galaxies (il les appelle "voies lactées»), qui contiendraient des systèmes planétaires parvenus à différents degrés de développement. Et faisant remarquer que ce niveau de l'activité cosmique "apparaît comme étant de nature éternelle et infinie par comparaison avec l'être humain, si fortement limité ${ }^{20}$, il lui semble non seulement nécessaire, mais même justifié "scientifiquement», de penser au-delà du connu. Sa phrase rappelle celle, bien connue, du Hamlet de Shakespeare : «il y a plus de choses dans le ciel et la terre que n'en peut concevoir notre philosophie». La nature est donc beaucoup plus complexe qu'on ne l'imagine. Sa complexité est l'œuvre d'un esprit créateur - Schubert est en effet profondément chrétien, bien qu'il n'utilise pas le mot «Dieu»-, mais celui d' "esprit vital créateur" (schaffender Lebensgeist) qui ne se repose jamais et qui trouve son accomplissement ultime dans la vie elle-même. Dans le cas de l'être humain, l'âme est la cause de la vie et elle agit dans toutes les parties du corps. Mais les corps individuels ne peuvent pas percevoir directement cette vie et encore moins son auteur, l'âme. Ils ne peuvent le faire qu'à travers des sensations concrètes, forcément partielles. La première chose ainsi ressentie est l'appartenance à la planète, à cause de la gravité qui, en attirant tous les êtres vers un point central, leur montre aussi la radicale unité de la planète. Mais la gravité constitue seulement un pôle, le négatif; son contraire, ou son complémentaire, le positif, se manifeste dans le magnétisme, qui éveille les sympathies entre les êtres ${ }^{21}$. Il ne s'agit plus alors d'une simple appartenance à une communauté physique mais de quelque chose de plus subtil : d'un lien qui, au-delà des créatures, nous unit à la totalité de la Création. On peut voir ici comment le «cœur» apparait de nouveau, comme chez Ritter, mais d'une façon plus tolérable pour les idées scientifiques de l'époque. Et le magnétisme - comme la gravité - apparait comme l'une des manières par lesquelles l'âme se fait présente à la vie qu' elle-même a créée.

Dans cette perspective le magnétisme animal serait semblable à ce "Kuss der ganzen Welt» - ce baiser du monde entier - que Beethoven chante dans sa neuvième symphonie. Pour notre auteur il en résulte, par une sorte d'ironie, que l'état magnétique entretient une profonde similitude avec la mort. Il ne faut pas penser qu'il s'agit, ici, du goût bien connu des romantiques pour le macabre et le deuil. C'est plutôt le contraire. Certes, en remarquant que les somnambules déclarent souvent se sentir éclairés par une lumière spéciale, comme depuis l'intérieur, sensation qui parfois s'accompagne de phénomènes qui, pour l'observateur, ressemblent à des évanouissements, et qui sont vécus par le sujet avec une impression de mort imminente, Schubert met en relief cette parenté du somnambulisme et de la mort. Et il ajoute que cette lumière intérieure rappelle la phosphorescence qui résulte de la putréfaction 
de la matière organique. Mais il faut ne pas oublier, rappelle-t-il aussi - que la putréfaction n'est qu'une sorte de combustion, et que la combustion est l'image archétypale de la transformation, c'est-à-dire de la vie au sens le plus large. C'est peut-être pour cela que ces évanouissements et ces sensations de mort imminente ont parfois la vertu de sauver de la mort des gens tenus pour incurables ${ }^{22}$. Voici la polarité et l'éternelle transformation si chères aux Naturphilosophen, qui font de la mort et de la vie des notions relatives, comme Goethe, d'une manière poétique et lapidaire - romantique! - l'avait formulé dans son célèbre vers "stirb und werde!» : «meurs et deviens!»

Cette perspective dynamique confere tout son intérêt au magnétisme aux yeux d'un romantique tel que Schubert. En ce qui concerne concrètement le corps du sujet, ce dynamisme et cette polarité se manifestent dans la dialectique, typiquement romantique, "ganglionnaire/cérébral» ${ }^{23}$. La partie physique du magnétisme consiste, selon Schubert, dans la stimulation de la force nerveuse du somnambule par le magnétiseur. Les passes commencent par la tête, comme si elles cherchaient à stimuler le cerveau pour que celui-ci diffuse leurs effets à toutes les parties du corps. Mais lorsque les différents organes se sont à leur tour éveillés, ils exercent aussi des actions sur le cerveau, qui se révèlent par l'apparition du sommeil magnétique ${ }^{24}$. Ces actions s'effectuent à travers le système nerveux végétatif, ganglionnaire, «multicentrique». Celui-ci constitue l'organe du Gemeingefühl- «sens commun» ou "sens général»-, une sorte de sens interne qui perçoit de manière unitaire les sensations les moins évidentes qui parviennent à notre organisme. Ainsi peut-on avoir des perceptions qui n'ont rien à voir avec celles des cinq sens classiques. Dans l'état de veille, soutient Schubert, le Gemü̈ ${ }^{25}$ se trouve non seulement impressionné par ce qui l'atteint à travers les sens corporels, particulièrement par la vue, mais aussi, d'une façon ininterrompue, par ce que le Gemeingefühl perçoit, bien que ce Gemeingefühl ne soit presque jamais accessible à la conscience. Cependant, dans l'état magnétique, les deux sortes de perceptions se recouvrent, et celles du Gemeingefühls'expriment d'une façon analogue à celles de la vue. Ainsi deviennent-elles accessibles à la conscience au point de se substituer aux perceptions communes.

C'est également en partant de l'omniprésente perspective du devenir, du Werden, que Schubert interprète les visions des somnambules non seulement comme des faits, mais comme de possibles annonces de l'inconnu. Parfois ces visions permettent d'imaginer ce qu'on pourrait observer «à travers les organes encore non formés d'un être plus élevé encore à venir» ${ }^{26}$. Il semble que, pour Schubert, cet homme du futur soit quelqu'un qui, plutôt que de

22. Idem, p. 357-358.

23. Voir le chapitre IV de Georges Gusdorf, Le romantisme..., ouv. cité, p. 228-246, intitulé « Ganglionnaire et cérébro-spinal».

24. Gotthilf Heinrich von Schubert, Ansichten..., ouv. cité, p. 361-362.

25. Gemüt désigne l'ensemble des fonctions sensitives et affectives de l'être humain.

26. Gotthilf Heinrich von Schubert, Ansichten..., ouv. cité, p. 363-364. 
développer des organes et des pouvoirs nouveaux, en aurait retrouvé quelques-uns déjà présents dans la nature, que nous aurions provisoirement perdus - ou, si l'on veut, qui seraient chez nous anesthésiés - lorsque nous avons donné la priorité à d'autres. Et, à ce propos, il s'immerge encore dans sa Nature vénérée et bien-aimée, pour nous rappeler qu'en matière de prédiction météorologique, par exemple, les meilleurs instruments n'ont pas atteint la précision que réalisent sans conscience quelques plantes - par exemple la Poriera hygrometrica - ou quelques animaux, surtout les insectes et parmi eux ceux qui sont asexués, ou qui ont peu développé la différenciation sexuelle, comme les fourmis ou les abeilles ${ }^{27}$.

La référence aux insectes, animaux considérés à l'époque comme inférieurs aux mammiferes et surtout à l'homme, doit être comprise dans la perspective de la théorie que Schubert va développer dans une ouvre postérieure, d'après laquelle le développement du cerveau, en augmentant la singularité des individus, les isole de la grande communauté de la nature et les rend aveugles et sourds à ses messages ${ }^{28}$. Mais elle permet également de revenir à l'être humain par une voie inattendue qui referme la boucle cosmique organisée autour du magnétisme animal. Les plantes et les insectes qui vivent une année

«[...] sont animés par le même esprit qui dirige les changements de la nature extérieure, et ils sont comme une image de l'année dans laquelle ils sont nés, et les périodes et les changements naturels qui se succèdent, les uns après les autres, pendant cette année, se développent aussi de manière autonome dans la brève vie de chaque chose. Ainsi chaque vie porte en elle-même le temps et le fondement de sa transformation, de la même façon que l'histoire externe de chaque être humain provient de son être intérieur. ${ }^{29}$

Nous voici de nouveau face au Kuss der ganzen Welt, le baiser du monde entier. Un baiser qui unit la science et la poésie, la matière et l'esprit, la conscience et l'inconscient. Le succès du magnétisme était garanti chez les romantiques. Mais, pour avoir le droit d'en user, les scientifiques et les médecins devront essayer de le légitimer face à ceux qui ne conçoivent pas le monde comme eux, ainsi que, dans le cas des meilleurs, face à eux-mêmes. Et cet effort va avoir des conséquences inattendues.

\section{Un NOUVEAU MALAde EXIGE DES NOUVELles MÉTHOdeS}

Cette entreprise de légitimation se déploie sur deux plans parallèles : le théorique et le pratique. Dans le premier, outre l'appui qu'ils prennent sur la

27. Idem, p. 365.

28. C'est la thèse qu'il soutient dans sa Symbolik des Traumes, ouv. cité.

29. Gotthilf Heinrich von Schubert, Ansichten..., ouv. cité, p. 368. 
philosophie de la nature de Schelling, les médecins qui pratiquent le magnétisme vont s'efforcer de concilier les intuitions propres à cette philosophie avec les doctrines physiologiques contemporaines. Ainsi fait Schubert lui-même, d'une manière exemplaire, dans le sixième chapitre de sa Symbolique du rêve, pour ne pas parler de son encyclopédique Histoire de l'âme. Et c'est aussi ce que font la plupart des auteurs qui vont vouer leurs efforts à la compréhension de la nouvelle doctrine. Mais l'un des traits les plus remarquables chez les principaux chercheurs du magnétisme animal en Allemagne est la façon d'étayer la théorie par des expériences pratiques. Carl Alexander Ferdinand Kluge (1782-1844), l'auteur de l'œuvre qui, en la matière, va jouir du plus grand succès, l'Essai d'une présentation du magnétisme animal comme moyen thérapeutique, déclare dans sa préface qu'il s'appuie surtout sur ses expériences personnelles ainsi que celles d'autres savants (il inclut dans ce champ celles de quelques magnétiseurs français), mais seulement si elles ont été strictement conduites de manière à ne pas verser dans la spéculation ${ }^{30}$. L'auteur le plus ambitieux, du point de vue intellectuel, parmi tous ceux qui réfléchissent sur le magnétisme, Dietrich Georg Kieser, n'arrivera à publier son Système du tellurisme ou magnétisme animal qu'après avoir consacré huit années et douze volumes de revue, l'Archive $d u$ magnétisme animal ${ }^{31}$, à l'analyse des écrits théoriques publiés en Europe ainsi qu'à la compilation de cas tirés de sources tenues pour fiables. C'est précisément cette dernière rubrique qui a suscité mon intérêt, car les cas très longuement détaillés publiés dans l'Archive, offrent une information extraordinaire sur la réalité de la pratique du magnétisme, ainsi que sur le monde, jusqu’alors presque inconnu, du malade.

Signalons à cet égard tout d'abord que, tout au long de la courte période de vigueur qu'a connue le magnétisme animal en Allemagne, le médecin a peu à peu perdu son rôle dominant, supplanté qu'il était par la somnambule $^{32}$. Cela ne s'est pas toujours produit, et on ne peut pas non plus affirmer catégoriquement qu'il s'agisse d'un phénomène qui se soit développé avec le temps, car il dépend surtout des personnalités respectives du magnétiseur et du malade. Il faut en tout cas prendre en compte que le magnétisme animal, en raison de son essence propre, tend à estomper la frontière qui sépare traditionnellement médecin et malade. Mais je crois pouvoir soutenir - et ce sera l'objet des pages qui suivent - que ce qui surgit au siècle des Lumières comme une pratique thérapeutique dirigée par le médecin se transforme pendant la période romantique en quelque chose de très hétérodoxe du point de vue professionnel, parvenant dans bien des cas à une inversion presque totale des rôles.

30. Carl Alexander Ferdinand Kluge, Versuch einer Darstellung des animalischen Magnetismus als Heilmittel, Wien, Franz Haas, p. III-IX.

31. Dietrich Georg Kieser, Archiv für den thierischen Magnetismus, 1817-1824, 12 volumes; System des Tellurismus oder thierischer Magnetismus, Leipzig, Herbig, volume I, 1822, volume II, 1826.

32. La quasi-totalité des cas présentés dans l'Archive est composée de femmes, et tous les cas étudiés sont des femmes. 
Avant même le développement du magnétisme dans l'esprit de la Naturphilosophie de Schelling, les partisans de cette nouvelle thérapeutique avaient observé, étonnés, que leurs patients, généralement des jeunes filles, manifestaient lorsqu'elles se trouvaient mises en état magnétique une transformation de leur personnalité qui n'avait rien de méprisable à leurs yeux : «Il ne reste rien en elles de l'affectation [...] et de ces autres limitations que l'étiquette, les préjudices et l'éducation imposent habituellement aux filles dans leurs relations avec le sexe masculin et qui barrent leurs expansions animiques. » ${ }^{33}$

Ce fragment montre de manière parfaitement claire le côté psychologique inconscient mis en lumière par le magnétisme animal : libérées de leurs inhibitions, les patientes deviennent plus authentiques dans l'expression de leurs pensées et de leurs opinions. Mais aussi, dans cet état, elles deviennent - comme l'auteur le remarque - capables de découvrir «les couleurs et le sens [des phrases écrites sur] des papiers mis sur leurs mains». Questionnée à ce sujet par son médecin, Arnold Wienholt, la première somnambule «historique» d'Allemagne, la jeune Catherine Albers, répondit : «- Je le vois. - Mais vous avez les yeux fermés. - Malgré tout je le vois. - Et comment le voyezvous? - Comme l'on voit dans le sommeil. ${ }^{34}$

Ce double versant, psychologique et parapsychologique, s'observe aussi dans les cas publiés par Eberhard Gmelin, un autre des grands magnétiseurs d'avant le romantisme. Deux de ses somnambules sont restées dans l'Histoire, non dans celle du magnétisme animal mais, plus précisément, dans l'histoire de la psychologie. La première d'entre elles fut incluse par Henri F. Ellenberger dans sa préhistoire de la psychiatrie dynamique, lorsque l'historien canadien reconnut dans son cas le premier dédoublement de la personnalité décrit et soigné médicalement ${ }^{35}$. La deuxième devint fameuse grâce à l'histoire de la littérature, qui a voulu voir en elle le modèle naturel utilisé par Heinrich von Kleist pour la protagoniste de sa nouvelle romantique Kätchen von Heilbronn $(1810)^{36}$.

La recherche historico-littéraire a refusé cette dernière hypothèse. Il est probable cependant que l'intérêt suscité par l'œuvre de Gmelin, en réalité assez faible, ait été stimulé par ce que les historiens de la littérature nomment la «légende». Mais d'un autre côté cette dernière est très significative, car elle révèle la troublante apparition d'une partie du psychisme dont on n'avait rien voulu savoir et de quelques présences inattendues : celle d'une femme qui n'était pas ce qu'on avait pensé, celle d'une femme différente de celle que la représentation traditionnelle donnait à voir. Apparaissait ainsi un type de femme qui détonnait par son autonomie psychique et ses capacités de révolte.

33. Alfred John, Thierischer Magnetismus und Schulmedizin in Bremen während der Aufklärung, Frankfurt, Peter Lang, 1997, p. 103.

34. Cité ibidem.

35. Henri F. Ellenberger, À la découverte de l'inconscient..., ouv. cité.

36. Gerhard Bauer, Eberhard Gmelin (1751-1809). Sein Leben und sein Werk, Heilbronn, Stadtarchiv Heilbronn, 1994, p. 46-61. 
L'œuvre de Kleist, et pas seulement dans le texte mentionné, en fournit un témoignage évident : dans La Marquise d'O., ainsi que dans Le prince Friedrich von Homburg, certains états qu'on pourrait nommer somnambuliques jouent le rôle de dei ex machina.

Il y a donc quelque chose qui bouge aux alentours - c'est-à-dire en dehors - de la médecine et qui tient au magnétisme animal et aux phénomènes qu'il provoque. Dans le cadre plus restreint de la relation thérapeutique c'est encore le médecin qui dirige le traitement. Mais, au vu de ces surprenants phénomènes, il commence déjà à ne plus opposer de résistance à se laisser guider par sa somnambule, qui elle-même commence à disposer d'une autonomie qui lui était interdite jusqu'alors, comme malade et comme femme, dans sa relation au médecin. Des médecins instruits dans l'esprit des Lumières se trouvent ainsi, grâce au magnétisme animal, face à des patientes qui, en vertu de leur condition de somnambules, sont déjà des romantiques, dans la mesure où elles luttent pour transmuter leurs "vapeurs" passives en tentatives de rébellion et d'auto-affirmation. Il ne se passera pas beaucoup de temps avant que ces tentatives ne se muent en assauts subreptices contre l'autorité, jusqu'alors incontestée, du médecin.

\section{LA RÉBELLION SOMNAMBULIQUE}

De tous les cas que j'ai eu l'occasion d'étudier, celui qui selon moi marque le commencement de ce transfert partiel de pouvoir fut publié dans l'Archiv für den thierischen Magnetismus en 1819 par Carl Christian von Klein, célèbre chirurgien de Stuttgart, qui ne devait que sur le tard, pendant les dernières années de sa vie, s'intéresser au magnétisme. En 1818, Klein avait été appelé pour soigner par magnétisme une jeune fille de 17 ans, Lotte K., orpheline de mère. Le tableau symptomatique que présentait la patiente était semblable à la majorité de ceux qui étaient décrits dans le milieu magnétique : aménorrhée, crampes et convulsions et parfois éclatantes crises de dyspnée et d'apnée qui faisaient craindre pour sa vie. Bien que le journal du traitement magnétique qui constitue la presque totalité du document semble dans son ensemble ne témoigner que du rapport traditionnel du malade au médecin, au cours de la thérapie, la jeune fille se permet quelques «libertés» assez choquantes pour l'époque, non seulement avec le traitement mais surtout à l'égard du docteur Klein.

À vrai dire, l'intervention de la malade dans son traitement n'est pas tellement surprenante, puisque l'une des capacités mises au jour par le magnétisme animal réside justement dans la clairvoyance des patients quant au diagnostic de leur mal et à son traitement. Cependant, quelques remarques faites par la jeune fille durent sembler étranges à un médecin aussi reconnu et expérimenté que Klein. Ainsi, par exemple, lorsqu’elle demande qu’on lui 
administre un médicament contre les vers intestinaux en insistant pour qu'on le fasse lors du dernier quartier de la lune, et qu'elle accompagne sa prescription d'une phrase provocatrice : «vous les médecins pouvez le croire ou non : cela aide plus avec le dernier quartier ${ }^{37}$; ou bien lorsqu'elle introduit dans le traitement la dégustation de friandises, comme elle l'indique, pendant le sommeil magnétique, à son thérapeute :

"Quand le temps s'améliore tu dois me dire pendant l'état de veille que je dois prendre un bain à neuf heures et demie du matin, en restant une demie heure dans le bain, et après boire une tasse de chocolat avec une cuillerée de fleur d'oranger pulvérisée $[\ldots]$ et que je peux tremper dans le chocolat tout ce que je voudrai.» ${ }^{38}$

Il est aisé de penser que mademoiselle Lotte est une manipulatrice; et elle l'est sans doute, comme les autres malades dont il va être question ci-dessous. Mais celle-ci et celles-là payent chèrement cette manipulation, car il est inconcevable qu'un médecin expérimenté puisse confondre une attitude purement théâtrale avec les vrais symptômes d'une maladie, même si cette maladie n'a rien de fondamentalement somatique. Et, comme je l'ai déjà remarqué, dans le cas qui nous occupe, la manipulation - et avec elle quelque chose qui a à voir avec le sens profond de la maladie et de son traitement ne s'arrête pas aux aspects purement techniques : elle dérive vers la sphère sentimentale où il faut chercher les racines de la maladie de Lotte K. On peut avancer que cette maladie sera totalement guérie à la fin du traitement magnétique.

Dans ladite sphère on peut situer aussi la façon particulière dont Lotte demande qu'on lui donne du vin dans un but thérapeutique. La prescription est bien connue à l'époque, mais la jeune fille assure que pour que le vin exerce son effet Klein doit boire d'abord, et le médecin observe que la fille boit toujours en appuyant ses lèvres au même endroit du verre que lui ${ }^{39}$. Si ce petit détail évoque le flirt, un autre portera encore plus loin les prétentions de la malade. À l'occasion d'une absence qu'elle a elle-même provoquée en se prescrivant des bains à Maulbronn, elle propose, pour ne pas rompre le lien magnétique avec son thérapeute, qu'on forge deux anneaux de fer qu'ils doivent porter. Klein déclare dans son texte l'avoir porté parfois au doigt, parfois dans la poche de son gilet. Il semble qu'on passe du flirt aux fiançailles, et même - qui sait? - au mariage, car il se trouve que Klein est déjà marié, mais que curieusement - en contradiction avec les observations de Gmelin et de

37. Carl Christian von Klein, «Geschichte einer durch Magnetismus in 27 Tagen bewirkten Heilung eines 15 monatlichen Nervenleidens", dans Archiv für den thierischen Magnetismus, volume 5 (1), 1819, p. 61.

38. Idem, p. 90-91.

39. Idem, p. 80. 
plusieurs autres auteurs - c'est l'or, et non le fer, qui provoque troubles et souffrances chez la malade, ce qui oblige le médecin à quitter son anneau de marié pendant la thérapie ${ }^{40}$.

La lecture détaillée de ce cas montre très clairement qui tient les rênes de la thérapie, en obligeant même le thérapeute à mettre le pied sur des sables mouvants, dangereux au moins pour sa respectabilité. Quoi qu'il en soit, cette histoire présente une singularité qui permet de mieux comprendre pourquoi le médecin se prête à tout ce que désire sa capricieuse malade. Cette singularité apparaît à travers quelques rares passages quasiment dissimulés au sein de la forêt de données que constitue le journal de la cure : Klein voudrait avant tout savoir si sa somnambule a la capacité de diagnostiquer et de prescrire des traitements à d'autres personnes, car son épouse est malade et la médecine est impuissante à soulager son mal ${ }^{41}$.

Les deux cas qui suivent procèdent de la romanesque collection rassemblée par Justinus Kerner, médecin de campagne qui, précisément grâce à ceux-ci et à d'autres du même genre, atteignit une grande notoriété en Allemagne et même à l'étranger. Le premier d'entre eux est sans doute le plus connu et le plus marquant : celui de Friederike Hauffe, «la voyante de Prevorst» ${ }^{42}$. Friederike était une jeune paysanne qui, au moment de ses fiançailles, tomba victime d'une inexplicable maladie aux symptômes somatiques et psychiques, dont des hallucinations qui en étaient la manifestation la plus remarquable. Pendant son enfance elle avait déjà eu quelques visions d'esprits qui avaient provoqué une certaine inquiétude chez sa famille, mais qui avaient finalement été tenues pour anecdotiques. Cependant, au cours de sa maladie, et surtout dès le moment où l'on décida de demander l'aide de Kerner, magnétiseur reconnu, ces visions devinrent plus fréquentes et surprenantes, car la voyante en arriva à être en communication avec un "monde d'esprits» doué d'une langue et d'un alphabet propres, dont Kerner n'a jamais douté. Certains ont considéré le cas de la voyante de Prevorst comme l'acte de naissance du spiritisme, non pas qu'il ait alors vraiment émergé comme phénomène de masse, mais parce qu'une théorie sur les esprits apparaissait ainsi dans l'œuvre d'un scientifique.

Cependant ce qui nous intéresse le plus ici c'est que le médecin, qui essaye d'imposer ses règles au début du traitement, tombe rapidement sous l'empire de la voyante, se bornant à agir en auditeur et transmetteur de ses révélations, aussi bien pour ce qui concerne la thérapie de sa maladie que pour l'approfondissement de la connaissance de ce monde parallèle des esprits. Dans le premier cas, Kerner va admettre l'usage des prières et des amulettes inventées par sa malade, et il va la magnétiser quand elle le sollicite, bien que

40. Idem, p. 77.

41. Idem, p. 140-141.

42. Justinus Kerner, Die Seherin von Prevorst, dans Werke, édition établie par Raimund Pissin, Hildesheim-Zürich-New York, Georg Olms, 1998, volume II, p. 13-245. La première édition date de 1829. J'ai étudié ce cas dans Luis Montiel, Daemoniaca. Curación mágica, posesión y profeća en el marco del magnetismo animal romántico, Barcelona, MRA, 2006, p. 37-62. 
souvent ce ne soit pas nécessaire, car Friederike tombe spontanément dans l'état magnétique. Il en va de même lorsque quelques malades accourent au chevet de la voyante, attirés par sa notoriété croissante. Kerner va constamment croire aux diagnostics de Friederike, diagnostics confirmés dans quelques cas par des expériences surprenantes. Par exemple lorsque l'épouse d'un pasteur vient lui demander une prescription pour une maladie hépatique, cette requête provoque la perte momentanée de la vue de l'un des yeux de la voyante. Surprise, la visiteuse reconnaît que, depuis quelque temps, elle a elle-même presque perdu la capacité de voir du même côté, mais qu'elle a gardé cette atteinte secrète. Il faut ajouter que ni le médecin, Kerner, ni l'époux de la dame ne s'en étaient aperçus jusqu'alors.

Ces diagnostics ainsi que les succès thérapeutiques obtenus poussèrent Kerner à abandonner son rôle dominant. Il fut particulièrement impressionné par la cure de la comtesse de Maldeghem qui, victime d'une maladie mentale, fut complètement guérie par les prières de la voyante.

Il faut évidemment avoir la crédulité, ou si l'on veut, la spiritualité d'un Justinus Kerner pour s'engager ainsi dans la "superstition». Mais dans la perspective de l'époque il faut admettre que Kerner s'appuie sur des phénomènes qui ont été observés par tous ceux qui se sont engagés dans la pratique magnétique : le magnétisme, et plus précisément ses somnambules, peuvent réussir, en matière thérapeutique, là où le médecin échoue.

Je citerai encore une autre voyante dont l'histoire est rapportée par Kerner, parce que son cas me permet soutenir la thèse suivante : grâce au magnétisme animal - et au préalable grâce à la maladie qui a provoqué son usage thérapeutique - quelques femmes arrivèrent à jouir d'un degré d'autonomie inconcevable pour l'époque et le milieu où elles vivaient. Elles façonnèrent leur propre vie, en le payant souvent très cher : la voyante de Prevorst, par exemple, mourut jeune, épuisée par l'anorexie et probablement par la tuberculose. Mais cela ne fut pas le cas de la protagoniste de cette deuxième histoire, Magdalene Grombach, «la fille d'Orlach » ${ }^{43}$.

Magdalene était originaire d'un petit village du Hohenlohe. Elle déclara, après l'apparition d'étranges phénomènes dans l'étable de la ferme familiale, y avoir été visitée par un esprit qui plus tard révéla qu'il était celui d'une nonne pécheresse de la Renaissance. La voyante, victime de convulsions résultant, d'après elle, de sa possession par un mauvais esprit (celui du moine séducteur de la nonne) fut traitée magnétiquement par Kerner, qui admit tout de suite l'origine surnaturelle de la maladie. En effet, depuis son expérience avec la voyante de Prevorst, il avait développé une théorie d'après laquelle c'était parce que le magnétisme animal donnait accès au monde des esprits qu’il était possible de soigner les maladies traditionnellement attribuées à la pos-

43. Comme dans le cas précédent cette histoire a été étudiée par moi dans Luis Montiel, Daemoniaca..., ouv. cité, p. 62-77. 
session démoniaque. L'histoire de la nonne et de son séducteur, telle qu'elle est racontée par Magdelene mise en état magnétique, est un vrai roman ou plutôt un mélodrame romantique, dans le sens le plus négatif du mot «mélodrame». Mais le plus intéressant - à mon point de vue - est que la guérison de la jeune fille, ainsi que la disparition des nombreux phénomènes paranormaux qui entouraient sa maladie, se produisirent seulement après que l'on eut obéi à l'ordre donné par l'esprit de la nonne : démolir la ferme et la reconstruire, - énorme travail sur le plan matériel et économique, mais aussi tâche pleine de sens dans une perspective psychologique profonde, comme effacement de l'ancien et renaissance à une nouvelle vie plus saine. Je soutiens qu'un tel bouleversement prouve qu'une personne socialement inférieure femme, adolescente, malade - est susceptible, grâce au magnétisme, de se faire valoir et d'imposer sa volonté à son père comme à son médecin.

Cependant l'exemple le plus extraordinaire, à mon avis, de cette dimension revendicative de la condition de somnambule nous est fourni par le cas, étudié par Kieser à partir de documents contemporains - car ce n'était pas sa malade - de «la jeune fille merveilleuse de Johanngeorgenstadt» ${ }^{44}$.

Il s'agit encore une fois d'une jeune femme (23 ans), fille d'un cordonnier qui était aussi le maire de la ville minière de Johanngeorgenstadt, en Saxe. Et encore une fois les symptômes consistent en une série de crampes et de convulsions qui conduisent à un état reconnu comme somnambulique par le médecin qui la traite. Pendant le cours de la maladie et de son traitement, cet état s'est teinté de symbolisme religieux à travers les discours prononcés par la malade, ainsi que grâce à une gestuelle facile à interpréter par les témoins des accès crisiaques. Bientôt quelques-uns devaient dire que c'était un ange ou l'esprit de Dieu qui parlait par sa bouche et annonçait «un nouvel Évangile du cœur». Ils affirmaient qu'ils n'avaient jamais rien entendu d'aussi beau et d'aussi convaincant sortir de la bouche d'un prêtre. À partir de ce moment la malade et son public semblent s'être réciproquement influencés : les postures qu'elle adopte la font comparer au Christ en croix, avec pour résultat la conviction que la jeune fille annonce sa propre crucifixion ${ }^{45}$.

La maison du maire devint alors un lieu de pèlerinage, car un nombre croissant des gens pensait qu'une nouvelle "Sauveuse», envoyée par Dieu lui-même, venait d'apparâtre. Et qui plus est, peut-être en signe prémonitoire d'une évolution à venir, plusieurs de ces pèlerins, et surtout nombre de femmes, crûrent qu'elle allait être la "Sauveuse des femmes " ${ }^{46}$. Dans cette situation, les autorités interdirent au maire cordonnier, sous peine d'emprisonnement, d'autoriser l'accès des pèlerins à sa maison. Les jours où l'on s'attendait à des crises, la maison était surveillée par des gardes, ce qui n'empêcha

44. Dietrich Georg Kieser, «Das wunderbare Mädchen in Johanngeorgenstadt», Archiv für den thierischen Magnetismus, 8 (1), 1820, p. 48-86.

45. Idem, p. 55-56.

46. Idem, p. 57-58. 
pas les gens d'accourir toujours aussi massivement. La situation devient extrêmement tendue, lorsque la jeune fille, pendant l'une de ses crises, annonça qu'au cours de la semaine de Pâques elle endurerait le supplice du Christ, que le vendredi elle serait crucifiée entre deux voleurs et prononcerait les mots qui furent ceux du Christ sur la croix, et que finalement elle demeurerait morte pendant trois jours, comme le Christ dans son tombeau.

Dans un climat d'attente exacerbée et malgré les efforts des autorités pour empêcher l'affluence du public, la «jeune fille merveilleuse» fit tout ce qu'elle avait annoncé. Ces événements amenèrent la prompte disparition de ses symptômes, disparition certifiée par le médecin, et apparemment garantie par le maintien ultérieur de sa bonne santé. Il me semble que, une fois réalisée cette prouesse que représenta la mise en scène de la passion de Christ, la jeune fille avait atteint un point difficile à dépasser et sûrement assez satisfaisant pour elle. Elle retourna, d'après le récit publié par Kieser, aux ouvrages de dentelle qu'elle avait dû abandonner à cause de sa "maladie magnétique», car les objets métalliques déclenchaient des convulsions. Mais celle qui revient ainsi à la vie «normale» n'est pas seulement Friederike Erdmuthe Reinhold, la fille du cordonnier, mais «la jeune fille merveilleuse de Johanngeorgenstad», et pour certains de ses contemporains "la Sauveuse des femmes».

\section{UNE RÉVOLUTION AVORTÉE}

Sa propre inconsistance théorique, liée à l'hostilité de la médecine officielle, finit par marginaliser la médecine magnétique dont les romantiques allemands avaient rêvé. Cependant, les "dossiers du magnétisme " ${ }^{47}$ conservés outre-Rhin fournissent aujourd'hui la preuve que la traditionnelle relation médecin-malade n'allait pas sans turbulences. Bien que masquées, celles-ci devaient se manifester encore bien des fois jusqu'à la rencontre de Freud avec les malades de Charcot. Et il faut ajouter que ces dossiers, si souvent réfléchis et pondérés, n'ont pas encore livré tous les éléments dont notre médecine contemporaine pourrait tirer bénéfice.

Luis Montiel est professeur titulaire d'histoire des sciences à l'Université Complutense de Madrid (Historia de la Ciencia, unidad de Historia de la Medicina, Facultad de Medicina ${ }^{48}$

47. Avec cette expression, Bertrand Méheust désigne la masse des faits que les critiques du magnétisme animal ont choisi d'oublier. Bertrand Méheust, Somnambulisme et médiumnité, Le Plessis-Robinson, Institut Synthélabo, 1999, vol 1, p. 41.

48. Je remercie Marie-Christine Pouchelle ainsi que Nicole Edelman pour leur minutieuse révision du manuscrit. 